\title{
Adaptive weighted morphology tree classifier for aircraft type recognition
}

\author{
Zhi-Gang Liu* and Yan-Ying Guo \\ Guangzhou Civil Aviation College, Guangzhou, Guangdong, China \\ E-mail: liuzhigangcauc@126.com \\ *Corresponding author
}

\begin{abstract}
Aircraft type recognition is a problem and key factor of safe docking of aircraft in airport auto-docking guide system. Based on adaptive weighted morphology tree classifier algorithm, it is approved for aircraft type recognition to solve the key issues to docking guide, useing image analysis and recognition technology to complete the type discrimination, so as to overcome the shortcomings of the aircraft in the whole range of berth travel and improve the speed and reliability of the search. According to the feature of target size and feature change during the process of aircraft movement, the adaptive weight morphological algorithm extracts the invariant features, which can solve the problem of feature transform of aircraft type recognition. The experimental results indicate that the effectiveness of the current recognition system is given. The system provides a good performance in aircraft recognition and offers better robustness against noise and poor image quality, which can satisfy the auto-docking system requirements of high precision, rapid speed and stabilization.
\end{abstract}

Keywords: Aircraft Type Recognition; Adaptive Weighted Morphology; Tree Classifier Least Distance; Auto-docking System.

\section{Introduction}

Moving target recognition is an important research area of pattern recognition, and has been widely used in agriculture and medical fields and so on. At the airport the automatic docking guidance system, in order to prevent the docking aircraft error and causing flight delays, or some special airplanes use specific berth must be of docking aircraft types for recognition. Identification of aircraft types is the key to the success of guidance system, and the current impact of the system application problems.

The difficulty for Aircraft types recognition lies in the target itself does not have a common gray feature, and different types of aircraft shape, size, gray difference is very large, so it is difficult to get the aircraft through the gray 
method complete and accurate appearance, it is difficult to distinguish the model, the recognition rate is low. At present, aircraft types recognition methods include: image matching, neural network recognition [1], invariant moment (or boundary moment) [2-3], Fourier descriptor [4-5] or geometric in-variants ${ }^{[6]}$. The algorithms are characterized by the global profile, and the three methods are essentially based on the assumption that the complete and accurate contour of the object can be obtained. Therefore, the use of the overall characteristics of the outline has its shortcomings, and extracted from the outline of some good robustness, easy to extract and sufficient to determine the key parameters of the target, rather than the entire profile as the feature, will have a better recognition effect.

\section{Feature Representation and Recognition of Plane Image}

A significantly different characteristics in different types of aircraft wingspan, length, engine number, to effectively identify the scale, size and rotation of the plane, with the above conditions do not occur transform features. This paper selects the ratio of length and wingspan, wingspan and altitude ratio, engine number, cockpit window shape and escape door, main landing gear number features, the adaptive weighted morphology for translation, rotation and scale invariant of the main characteristics of the type recognition, the training sample database on the features of target performance analysis. Then tree classifier is used to classify and identify. The process is shown in Figure 1.

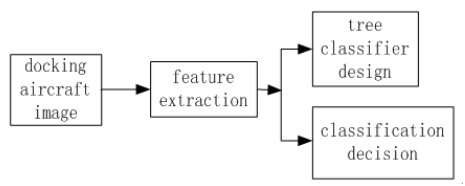

Fig. 1 Recognition procedure

\subsection{Feature extraction}

Feature extraction is related to the speed and accuracy of recognition and classification. In this paper, the performance of the target representation is better characterized by the adaptive weight morphology. Feature extraction is simple, less quantity, so that the classification function of the tree classifier is simple, easy to hardware implementation. Specific features are defined as follows:

(1) The ratio of length and wingspan obtain:

The plane's ratio of length and wingspan can be the following steps to

a) Determine the direction of the spindle: 


$$
\alpha=\frac{1}{2} \tan ^{-1} \frac{2 \mu_{11}}{\mu_{20}-\mu_{02}}
$$

For $\alpha$ is the angle between the principal axis and the horizontal direction, $\mu_{i j}$ is the center invariant moments of the image.

b) Rotate the image so that the principal axis is parallel to the horizontal direction.

c) The plane image is projected in two directions of horizontal and vertical, and obtained shadow of the horizontal $x^{\prime}$ and vertical directions ${ }^{y}$.

d) Ratio is $R=x^{\prime} / y^{\prime}$.

(2) The ratio of aircraft wingspan and altitude. $z$ is the height of the aircraft shadow, and ratio is $T=\frac{y^{\prime}}{z^{\prime}}$.

(3) Engine number, cockpit window shape and escape door, main landing gear number is extraction of adaptive weighted morphological algorithm.

The definition adaptive weighted corrosion (WER) and inflation (WDI) is as follows:

$$
\begin{aligned}
& W E R(k, l)=\min _{u, v}\{X(k+u, l+v) / B(u, v)\} \\
& W D I=\max _{u, v}\{X(k-u, l-v) \times B(u, v)\}
\end{aligned}
$$

For $B$ is structural element, $X$ is original image.

Structural element $B$ has standardized weighted factor and the values of factor are calculated by this method: The values of the edge direction of the weight is 1 , the weighted values of the farthest point are assigned in accordance with the weight factor $\omega>1$, This leads to a very important influence on the edge points and reduces the influence of adjacent points. The rest of the weight values are calculated based on $\Delta \omega=(\omega-1) / d$, and $d$ is the distance between the edge point and the distance from the edge point. In horizontal and vertical direction, weighted values in accordance with $\Delta \omega$ growth, each step starts from the edge point. For example, for the size of $3 \times 3$ of the structural elements $B$, if the horizontal direction of the edge $\omega_{1}=3$,so $B$ is $B_{1}$, which $\Delta \omega=2$, suppose that $\omega_{2}=3$, and the same edge points in the direction of the 45 degree, $B$ is $B_{2}$, which $\Delta \omega=1$.

$$
B_{1}=\left[\begin{array}{ccc}
-1 & -1 & -1 \\
1 & 1 & 1 \\
-1 & -1 & -1
\end{array}\right] \quad B_{2}=\left[\begin{array}{ccc}
\underline{1} & 0 & -1 \\
0 & 1 & 0 \\
-1 & 0 & 1
\end{array}\right]
$$




\subsection{Feature analysis}

In order to quantitatively describe the quality of the feature, each feature is regarded as a random variable which obeys Gauss distribution, and is described by its mean and standard deviation:

$$
\begin{gathered}
m=\sum_{i=1}^{N} f_{i} / N \\
\sigma=\sqrt{\sum_{i=1}^{N}\left(f_{i}-m\right)^{2} / N}
\end{gathered}
$$

According to the following equation:

$$
\left|m_{i}-m_{j}\right|>3\left|\sigma_{i}-\sigma_{j}\right|
$$

The characteristics of the plane sample target expression quality, determine the representation model of plane target. These analyses can also be used to determine the priority of classification features. In this paper, apply this method to sort the characteristic quantity according to the advantages and disadvantages, and then apply the superior features to classify, and then use the sub optimal feature to classify the next layer.

\subsection{Classification and recognition of tree classifier}

In this paper, the adaptive weighted morphology algorithm is embedded into the bottom of the tree classifier. The analysis of the extracted features, the classification tree is set up according to the advantages and disadvantages of the characteristic description. In the classification tree, cannot be characterized by a single better clear description of the other sub-optimum characteristic quantity of with the minimum distance method of clustering, so as to achieve the purpose of all target classification. Flow chart of algorithm is implemented as shown in figure 2 .

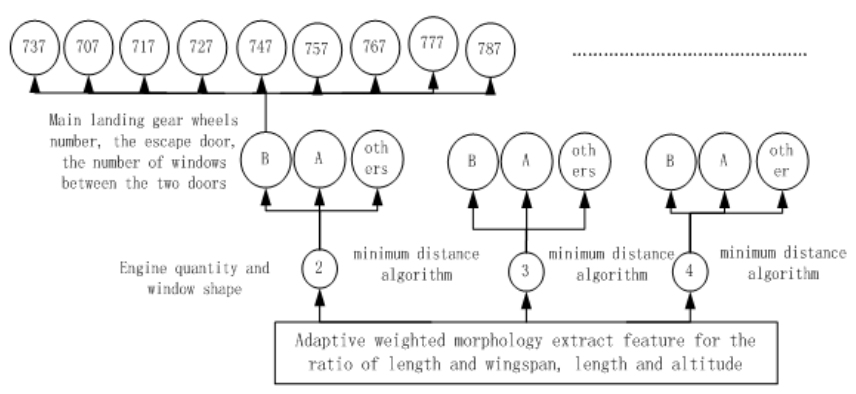

Fig. 2 Algorithmic flow chart 


\section{Experimental Result}

In this paper, the real video image of BaiYun airport aircraft berth is used, and a part of the video image is added to the noise. The two valued aircraft images of segmentation are used to identify, and the BP neural network is compared with the experimental results. Ten types of aircraft target 200 images (boeing737/747/777, airbus320/340/380, cessna172, IL86, MD82, CRJ200ER) train, At the same time, the noise is added to the image after 50 training, and then use the video image to test the berth. The experimental results are as follows:

The classification experiment of three types of docking aircraft image with less than 0.3 noise was carried out, for B777, A320, IL86, the results are shown in table 1 .

Tab. 1 Three different types noise aircraft recognition

\begin{tabular}{|c|c|c|c|}
\hline Recognition methods & $\begin{array}{c}\text { Minimum } \\
\text { distance }\end{array}$ & $\begin{array}{c}\text { BP neural } \\
\text { network }\end{array}$ & $\begin{array}{c}\text { Minimum } \\
\text { distance tree } \\
\text { classifier }\end{array}$ \\
\hline input images & 40 & 40 & 40 \\
\hline recognition accuracy & 34 & 37 & 39 \\
\hline recognition rate\% & 87.5 & 92.5 & 97.5 \\
\hline
\end{tabular}

\section{Conclusion}

In this paper, the algorithm uses adaptive weight morphology to extract features, and hierarchical input tree classifier is used to get the purpose of aircraft type's recognition. In which the characteristics of the aircraft are extracted by adaptive weighted morphology, which can solve the problem of aircraft matching, but also can remove the noise of the docking image, thus improve the accuracy and efficiency of the recognition and the ability of feature extraction and robust adaptation. Finally, the minimum distance tree classifier is used to solve the problem of the docking system, and a good result is achieved. So the research work in this paper has important theoretical significance and broadly application prospects in the intelligent traffic management.

\section{Acknowledgments}

This work was financially supported by the Guangdong Higher Vocational Education Teaching Reform Project (No.20130301051) and the training project 
for outstanding young teachers in higher education institutions of Guangdong Province(No. YQ2014177).

\section{References}

1. Syed Zafar Ali and Muhammad Ahmad Choudhry. A generalized higher order neural network for aircraft recognition in a video docking system [J]. Neural Computing \& Applications, 2010, Volume 19(1), 21-32.

2. M Breuers. Based planepose estimation using moment invariants[C]. In SPIE Conference on automatic target recognition IX. Orlando, Florida, 1999, 3718(4).

3. Yan-Ying Guo, Guo-Qing Yang; Li-Hui Jiang, Adaptive weighted morphology detection algorithm of plane object in docking guidance system [J]. International Journal of Advanced Robotic Systems, 2010, v 7, n 2, p 99-104.

4. Park, Jin-Yeong, Jun, Bong-huan et al. , Experiments on vision guided docking of an autonomous underwater vehicle using one camera [J], Ocean Engineering, 2009,v 36, n 1, p 48-61.

5. Feng Zhang,Shang-qian Liu,Da-bao Wang,Wei Guan. Aircraft recognition in infrared image using wavelet moment invariants [J]. Image and Vision Computing. 2008 (4).

6. Wang X F, Zhang X Y, Gao R N. Research of image edge detection based on mathematical morphology. International Journal of Signal Processing, Image Processing and Pattern Recognition. 2013. 